
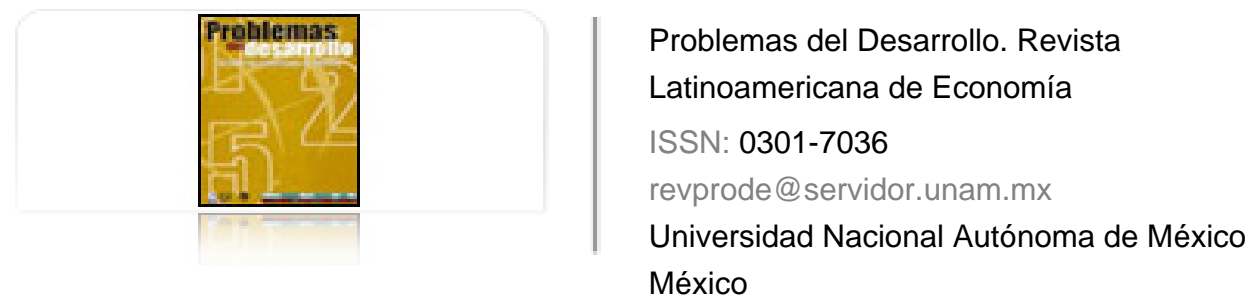

Bekerman, Martha; Cataife, Guido

Las microfinanzas en Argentina: teorías y experiencias

Problemas del Desarrollo. Revista Latinoamericana de Economía, vol. 35, núm. 136, 2004, pp. 115-

136

Universidad Nacional Autónoma de México

Distrito Federal, México

Disponible en: http://www.redalyc.org/articulo.oa?id=11825946007

Cómo citar el artículo

- Número completo

- Más información del artículo

Página de la revista en redalyc.org

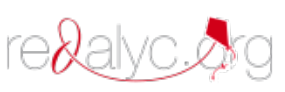

Sistema de Información Científica

Red de Revistas Científicas de América Latina, el Caribe, España y Portugal Proyecto académico sin fines de lucro, desarrollado bajo la iniciativa de acceso abierto 


\title{
Las microfinanZas en Argentina: teorías y eXPeriencias*
}

\author{
Martha Bekerman** \\ Guido Cataife***
}

Fecha de recepción: 22 de septiembre de 2003. Fecha de aprobación: 19 de mayo de 2004.

\section{Resumen}

El presente trabajo intenta introducir la temática del estado actual de las microfinanzas en Argentina. Para ello se combina el abordaje teórico con el práctico. El análisis teórico gira en torno a los conflictos entre sustentabilidad e impacto social, por un lado y sustentabilidad y escala por el otro, conceptos que se definen en el texto. El análisis empírico, por su parte, consta de una descripción de la situación de las microfinanzas en Argentina basada en los datos disponibles, más un estudio de campo. Este último, realizado a los efectos de comprender el impacto de los conflictos mencionados en la estructura de microfinanzas particular de Argentina.

Palabras clave: instituciones de micorfinanzas, impacto social, pobreza y equidad social, políticas públicas, impacto en la sustentabilidad.

\begin{abstract}
The paper is intended as an introduction to the subject of the present state of micro-financing in Argentina. For this purpose, it combines a theoretical with a practical approach. The theoretical analysis moves, in turn, to the conflicts between sustainability and social impact, on the one hand, and sustainability and scale, on the other, concepts that are defined in the text. The empirical analysis, for its part, involves a description of the state of micro-financing in Argentina based on the available data, plus a field study. The latter was carried out in order to understand the impact of the conflicts mentioned for the particular structure of micro-financing in Argentina.
\end{abstract}

Key terms: micro-financing institutions, social impact, poverty and social equity, public policies, impact on sustainability.

* Los autores agradecen la colaboración de Sabina Ozomek.

* Directora del Centro de Estudios de la Estructura Económica (censes) de la Facultad de Ciencias Económicas, Universidad de Buenos Aires. Correo electrónico: bekerman@econ.uba.ar

*** Investigador del Centro de Estudios de la Estructura Económica (cenes) de la Facultad de Ciencias Económicas, Universidad de Buenos Aires. Correo electrónico: gcataife@yahoo.com 


\section{Résumé}

Le présent article introduit la thématique de l'état actuel des micro-financements en Argentine et, dans ce but, fait une comparaison entre leur aspect théorique et leur aspect pratique. L'analyse théorique est axée sur les conflits entre le développement financier soutenu, la rentabilité à long terme et 1 'impact social, d'une part, et ces concepts tels que définis dans l'article et les niveaux structurels auxquels sont dirigés ces micro-crédits, d'autre part. L'analyse pratique, de son côté, comprend une description de la situation des micro-financements en Argentine basée sur les informations disponibles ainsi qu'une étude de données. Cette dernière a été faite afin de comprendre l'impact des conflits mentionnés plus haut sur la structure particulière des micro-financements dans ce pays.

Mots-clés: institutions de micro-financements, impact social, pauvreté et équité sociale, politiques publiques, impact sur la possibilité de développement financier soutenu et rentabilité à long terme.

\section{Resumo}

O presente artigo apresenta uma introdução à temática do estado atual das microfinanças na Argentina, através da combinação de abordagens teóricas e práticas. A análise teórica explora, por um lado, os conflitos entre sustentabilidade e impacto social, e por outro, sustentabilidade e escala, conceitos definidos no texto. A análise empírica se constitui de uma descrição da situação das microfinanças na Argentina, baseada nos dados disponíveis, além de um estudo de campo realizado com o intuito de compreender o impacto dos conflitos mencionados sobre a estrutura das microfinanças particulares da Argentina.

Palavras-chave: instituições de microfinanças, impacto social, pobreza e eqüidade social, políticas públicas, impacto na sustentabilidade. 


\section{Introducción}

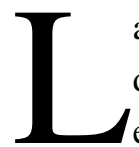

a extensión y profundidad de la crisis argentina reciente ha generado niveles de desempleo y precarización laboral que no encuentran precedentes históricos. En ese sentido podría señalarse que las transformaciones sociales de carácter negativose tradujeron en una mayor demanda por parte de los potenciales beneficiarios de microcréditos. Sin embargo, su desarrollo en Argentina muestra un carácter extremadamente incipiente, aún cuando se lo compara con otros países de América Latina.

Tabla 1

Microempresas en Latinoamérica

\begin{tabular}{lrc}
\hline \multicolumn{1}{c}{ País } & $\begin{array}{c}\text { Cantidad } \\
\text { de microempresas }\end{array}$ & $\begin{array}{c}\text { Microempresas con } \\
\text { crédito de IMFs (\%) }\end{array}$ \\
\hline Bolivia & 1362321 & 27.83 \\
Nicaragua & 41757 & 20.18 \\
El Salvador & 667186 & 14.06 \\
Honduras & 89118 & 12.01 \\
Chile & 1207184 & 6.86 \\
Guatemala & 1421714 & 5.01 \\
Costa Rica & 311219 & 4.11 \\
Ecuador & 1694663 & 3.88 \\
República Dominicana & 1392188 & 3.55 \\
Colombia & 6501805 & 3.37 \\
Paraguay & 987326 & 3.06 \\
Perú & 6866193 & 2.7 \\
Panamá & 289004 & 2.21 \\
México & 10273945 & 0.65 \\
Uruguay & 341909 & 0.47 \\
Brasil & 18989753 & 0.33 \\
Argentina & 1911170 & 0.26 \\
Venezuela & 3247271 & 0.07 \\
\hline
\end{tabular}

Fuente: BID (Westley, 2001).

Este trabajo se propone avanzar en el conocimiento de las experiencias y potencialidades del sector de microfinanzas en Argentina, comprender las razones de su limitado crecimiento y evaluar sus requerimientos para expandirse y su potencial para combatir la pobreza.

Para ello se estudiarán las especificidades que presenta Argentina para el desarrollo de las microfinanzas y se presentarán los resultados de un trabajo de campo realizado en 
quince instituciones de microfinanzas (IMFS) pertenecientes a distintas zonas de dicho país. Es necesario aclarar que el señalado carácter incipiente del sector ha determinado la existencia de distintos tipos de dificultades para llevar adelante el análisis. En particular, podemos mencionar la carencia de estadísticas globales, la resistencia de algunas IMFS a brindar la información requerida, la falta de indicadores homogéneos, la inexistencia de estudios globales previos, ${ }^{1}$ entre otros.

Comenzaremos por un análisis sobre el significado de las microfinanzas, su relevancia en términos de eficiencia económica y equidad social, y una discusión sobre los objetivos de las IMFS. Posteriormente se analiza la situación de las microfinanzas en Argentina tomando en cuenta las especificidades que ofrece el contexto social e institucional. Más adelante se presentan los resultados del trabajo de campo. Finalmente, se plantean las conclusiones del trabajo.

\section{¿Qué son las microfinanzas y cuáles son sus objetivos?}

\section{Definición de microfinanzas}

No parece existir un consenso claro acerca del significado de las microfinanzas. El reconocido texto de Ledgerwood (1999) ofrece una definición estándar: "se refiere a la prestación de servicios financieros a clientes de bajos ingresos, incluyendo a los autoempleados". Para Rahman (2000) las microfinanzas constituyen un conjunto de "mecanismos innovadores para la provisión de crédito y de facilidades de ahorro destinados a aquella porción de la población tradicionalmente excluida del sector financiero formal".

Esa exclusión suele producirse porque los sectores de bajos ingresos presentan las siguientes características:

- No pueden ofrecer garantías aceptables

- Presentan un alto riesgo crediticio

- El monto de los créditos requeridos es demasiado pequeño para una institución que aspira a ser rentable.

Esto nos plantea la necesidad de encontrar respuestas a las fallas que presenta el mercado financiero: "Las microfinanzas intentan combatir la pobreza y desarrollar la capacidad institucional de los sistemas financieros, descubriendo diversas maneras de prestar (con bajos costos) a hogares carenciados" (Morduch, 1996). En ese sentido, Rahman (2000) afirma que tanto el crecimiento del sistema de microcréditos como el trabajo pionero de las organizaciones no gubernamentales (ONGS) han demostrado no sólo que los pobres no poseen un alto riesgo crediticio, sino que, además, están dotados de un gran potencial de ahorro.

1 Los estudios publicados en Argentina hasta la fecha corresponden a casos de instituciones individuales, como Carbonetto et al., (1996) Carbonetto et al. (2001), Lescanne (1998), pero no se orientan a dar una visión del sector en su conjunto. 
La definición de microfinanzas que se considerará en este trabajo incorpora los conceptos señalados e incluye dos más. Por un lado, el acceso a los servicios financieros de personas carenciadas y excluidas del sector financiero formal. Se incluye entonces a aquellas instituciones que brindan créditos de bajo monto, es decir, suficientemente pequeños que no los ofrece la banca formal. Por otro lado, incluye esquemas especialmente diseñados y mecanismos innovadores para la provisión de esos créditos de la manera más eficiente posible. Además de otorgar este servicio, muchas instituciones promueven el ahorro de sus clientes.

\section{Dos principios: eficiencia y equidad}

La existencia de las microfinanzas puede ser justificada desde dos perspectivas: la de mejorar la eficiencia y la de incrementar la equidad.

El tema de la eficiencia está inextricablemente ligado a las ya mencionadas fallas de mercado, es decir, al hecho de que éste no funciona en la práctica tan bien como en los modelos ideales de competencia perfecta. Esto hace que el sistema bancario no satisfaga la demanda de crédito de una cantidad de personas que, de tener acceso al mismo, estarían en condiciones de generar suficiente riqueza como para devolver el capital prestado, con su costo de oportunidad, y de mejorar su condición de vida. El que existan personas que potencialmente sean buenos prestatarios y que no reciban la oportunidad indica que estamos ante un problema de eficiencia: el mercado no provee los mecanismos adecuados para canalizar todas las transacciones que los agentes desearían.

El tema de la equidad está dentro de la función de preferencia de los ciudadanos junto con objetivos sociales diversos, tales como: reducir la cantidad de individuos que viven por debajo de la línea de la pobreza, dar trabajo a los desempleados, entre otros. Dos alternativas se abren aquí. En primer lugar, el desarrollo de emprendimientos privados espontáneos a partir de ciudadanos que se compenetran ideológicamente con estas causas y pueden llegar a constituir organizaciones no gubernamentales (ONGS). En segundo lugar, que los objetivos señalados lleguen a ser considerarlos bienes públicos, es decir, bienes que benefician a toda la sociedad. Es aquí cuando la provisión de equidad y justicia social a través de microcréditos se transforma en una responsabilidad del Estado que debería ser implantada a través de sus propias instituciones.

\section{El circuito de financiamiento de las microfinanzas}

A pesar de que las IMFS se proponen aumentar la equidad, no se comportan como meras instituciones asistenciales sino que otorgan créditos sujetos a devolución y al pago de tasas de interés que en algunos casos son relativamente altas con respecto a las vigentes en el mercado. ${ }^{2}$ De este modo, la secuencia del circuito monetario es: 1) las IMFS otorgan crédi- 
tos a los microempresarios; 2) éstos los usan para comprar o producir bienes o servicios que venden a otros microempresarios o a terceros; 3) con el dinero obtenido en esas ventas, los microempresarios devuelven sus créditos con los respectivos intereses a las IMFS que, en muchos casos, llegan así a autofinanciarse.

En algunos países, las IMFS reciben también ahorros de los microempresarios o de terceros, lo que puede ayudarlas a expandir sus créditos a un círculo mayor de la población carencial. En el caso de no alcanzar la autosustentabilidad, o como forma de iniciar sus actividades, las IMFs pueden recibir fondos de donantes privados o públicos.

Pero la secuencia de los flujos financieros que se da en este contexto es muy distinta a la planteada por la teorías de los circuitos monetarios o circuitistas. A partir del principio de la demanda efectiva de Keynes, estas teorías destacan el papel de la demanda de las firmas en la expansión del crédito bancario, ya que en respuesta a la misma, los bancos demandan, a su vez, depósitos de las familias ofreciendo a cambio una tasa de interés. El equilibrio de esta última queda determinado de tal modo que la cantidad prestada por las familias se iguale a la cantidad demandada por las empresas (Parguez y Seccareccia, 2000; Schmitt, 1996).

En el caso de las IMFS, son estas instituciones las que incentivan o inducen la solicitud de crédito por parte de los prestatarios ya que éstos nunca han tenido por su fuerte racionamiento. En muchos casos, los microempresarios pueden, incluso, carecer de una cultura ligada a la obtención del crédito, que debe ser desarrollada por las IMFs. Por otro lado, podría señalarse que el monto de dinero captado por estas entidades puede llegar a ser una variable exógena (sobre todo cuando fue otorgada inicialmente por donantes) y que no se ajusta en función de la demanda de créditos por parte de los microempresarios. Asimismo, la tasa de interés no se determina por la anulación de la demanda neta, pues como fue señalado anteriormente, distintas IMFS adoptan distintos criterios para fijarla.

\section{La polémica en torno de los objetivos de las IMFS}

Dentro de las instituciones de microfinanzas existe una gran divergencia de opiniones acerca de si el énfasis debe ser puesto en la sustentabilidad financiera o en la profundización del impacto social a través de la reducción de la pobreza. En ese contexto se discute particularmente cuál es el impacto en la sustentabilidad financiera al prestar a los sectores más pobres.

Desde el punto de vista del impacto social, la polémica se plantea con respecto a cuál es la estrategia que reducen en mayor medida de la pobreza: concentrarse exclusivamente en los más pobres o incluir a personas de bajos ingresos en general. A continuación desarrollaremos estos aspectos.

2 El nivel de las tasas de interés está ligado al grado en que las imfs se proponen alcanzar la sustentabilidad financiera, lo cual se discutirá en las próximas secciones. 


\section{El trade off entre impacto social y autosustentabilidad}

Los donantes internacionales, que representan una de las fuentes más importantes de provisión de fondos para las ongs que otorgan microcréditos, ${ }^{3}$ están imponiendo criterios muy estrictos de composición de capital propio con relación a los fondos recibidos, así como el creciente reclamo de que las mismas alcancen la sustentabilidad financiera.

Los argumentos esgrimidos en favor de la búsqueda de sustentabilidad financiera se vinculan con la necesidad de que las IMFS aseguren su perdurabilidad (a través de la independencia económica) con el tiempo, ya que solo así pueden llegar a alcanzar los objetivos propuestos. A esta razón podría añadirse otra más, relacionada con efectos dinámicos. Si consideramos la importancia del aprendizaje que las IMFs desarrollan con el paso del tiempo (especialmente en cuanto a las mejoras organizacionales que suelen producirse), la perduración se torna una condición necesaria para alcanzar un alto nivel de eficiencia. Asimismo, la meta de autosustentabilidad tendería a incrementar la eficiencia y a disciplinar el gerenciamiento de las IMFs.

La búsqueda de autosustentabilidad financiera puede llegar a producir cambios organizacionales y metodológicos en las IMFs que lleven a desvirtuar su objetivo inicial de aliviar la pobreza. En primer lugar puede implicar una redefinición de la población beneficiada hacia los estratos menos pobres dentro de los pobres y un aumento en el monto de los créditos (lo cual también puede tender a desplazar a los más paupérrimos). En segundo término, puede llevar a incrementar significativamente las tasas de interés de los préstamos para la gente necesitada. Esto equivale a un aumento en la proporción de la nueva riqueza generada por los clientes que las instituciones captan, por lo que el impacto sobre la pobreza tiende a reducirse.

\section{El trade off entre alcance $y$ autosustentabilidad}

El término alcance alude a la profundidad con que los microcréditos calan en la lucha contra la pobreza, es decir, al nivel de ésta al cual llegan los préstamos. El concepto escala, en cambio, se refiere a la cantidad de individuos que tienen acceso a los microcréditos, independientemente de su posición en dicho nivel.

Existe una discusión en torno al trade off entre alcance y escala. La misma se basa en el hecho de que asistir a los más pobres entre los pobres, si bien tiene un mayor impacto sobre la pobreza, puede estar asociado con un menor nivel de repago y con un mayor gasto operativo por préstamo. Inversamente, otorgar a los menos pobres, si bien impacta

Estos fondos sólo están dirigidos a una porción muy selecta de ongs, mientras que el resto, que por alguna razón no califican (escala, etapa de madurez, ubicación, enfoque, entre otros), quedan libradas a la obtención de sus propios recursos. 
menos sobre la pobreza extrema, podría presentar mejores expectativas de recuperación de los préstamos y de extensión de los mismos a un número mayor de microempresarios.

\section{Impacto social. El trade-off entre alcance y escala}

Cuando las IMFS adoptan una posición respecto a extender el alcance o la escala, explícita o implícitamente están asumiendo un coeficiente de ponderadores relativos de impacto de los microcréditos sobre los distintos niveles de pobreza. A partir de allí, queda automáticamente derivada la estrategia óptima para la IMF, es decir, la combinación alcance-escala que maximiza su objetivo. Consideremos el siguiente ejemplo: una IMF genera un impacto mayor prestándole a una persona muy pobre que a un individuo no tan pobre. Pero, a su vez, los costos operativos de otorgarle al primero son mayores que los del segundo. Nos preguntamos entonces cuál será la táctica óptima para la IMF. Pues bien, a ésta le va a convenir destinar sus fondos de modo tal que se maximice con ellos el impacto sobre la pobreza. Por ende, la estrategia óptima de la IMF dependerá de cuánto sea el diferencial de impacto (entre prestarle a un muy pobre y prestarle a uno no tan pobre) y cuánto sea el diferencial de costos operativos entre ambos.

\section{Las microfinanzas en Argentina}

\section{Su desarrollo en la zona rural}

Las microfinanzas han tenido un desarrollo muy pobre en las zonas rurales de Argentina, lo cual podría vincularse con las diferencias que presentan estas zonas con las de aquellos países donde las microfinanzas rurales tuvieron un amplio desarrollo.

Entre los factores más importantes que presenta la zona rural argentina, vinculados con el desarrollo de las microfinanzas, se encuentran los siguientes:

- Factor demográfico: la dispersión de la población rural de Argentina asociada a las grandes distancias que caracterizan a nuestro país hace que el costo operativo de las IMFS sea mucho más elevado que en otras regiones del mundo, mucho más concentradas demográficamente, como Bangladesh e Indonesia. Esto se debe a que las microfinanzas tienen, por su naturaleza, costos significativos asociados a las visitas que los agentes de crédito deben hacer a los clientes.

Sin embargo, según la Secretaría de Agricultura, Ganadería y Pesca (SAgyp), existen en el país alrededor de 180000 productores minifundistas, localizados en su mayoría en la zona extra-pampeana, ${ }^{4}$ que presentan una fuerte escasez de factores productivos

$4 \quad$ Las actividades productivas de estos productores pueden detallarse, a grandes rasgos, del siguiente modo: en el NEA, algodón, tabaco, yerba mate, mandioca, horticultura y ganadería vacuna. En el NOA, caña de azúcar, tabaco, algodón, especias, ovinos, caprinos y camélidos; en Cuyo, vitivinicultura, 
(tierra y capital) y un bajo nivel de absorción tecnológica, lo cual los coloca en una escala productiva subóptima, les genera un excedente productivo pequeño y un prácticamente nulo poder de comercialización. Es en algunas de estas regiones de minifundio en donde se vuelve aplicable el desarrollo de pequeños créditos rurales.

- Factor cultural: la tradición de crédito subsidiado creada por la banca pública. Schreiner (2000) señala que en el pasado los bancos públicos otorgaron préstamos fuertemente guiados por criterios de clientelismo político, lo cual complicaba su devolución. Esto significa que la experiencia de esos bancos en las zonas rurales ha generado una pérdida de la cultura de cumplimiento de pago y una identificación del crédito con los subsidios. A ello se debe añadir el que los préstamos en épocas altamente inflacionarias fueron otorgados a tasas que resultaron fuertemente negativas en términos reales, lo cual incrementó la concepción del préstamo como una ayuda.

Estos factores llevaron a Schreiner (2000) a concluir que, dada la tecnología actual disponible para manejar IMFs, la zona rural argentina no presenta, en general, las condiciones adecuadas para que tenga lugar un crecimiento significativo de las microfinanzas. Esta afirmación es compartida por Gonzalez-Vega (1998), para quien será necesario “esperar por otras innovaciones, posiblemente ligadas al desarrollo de las instituciones que gobiernen las garantías, los derechos de propiedad, la seguridad de los contratos y los procesos legales y judiciales". 5

\section{Las microfinanzas en la zona urbana}

A diferencia de lo que ocurre en la zona rural, la zona urbana de Argentina parece presentar todas las condiciones necesarias para que las microfinanzas se difundan ampliamente. En primer lugar, las condiciones del mercado crediticio son excesivamente restrictivas. ${ }^{6}$ En segundo término, se produjo una ampliación del mercado para las microempresas. La marginalidad social está actualmente tan difundida que existe una aceptación mucho mayor por parte de la población para comprar bienes o contratar servicios de microempresas, usualmente más accesibles que las marcas comerciales.

No obstante estas situación favorable que presenta la zona urbana, existen algunos factores que limitan fuertemente el desarrollo de las microfinanzas. Entre ellos podemos señalar, por un lado, la escasa participación del estado en la implantación y promoción de la mismas y la existencia de regulaciones que actúan en contra de su desarrollo. Y, por el otro, el vertiginoso auge alcanzado por el trueque durante los años recientes.

frutihorticultura y caprinos, en la Patagonia, ovinos y caprinos; en el Centro, tambo, caprinos, avicultura, vacunos para cría y cereales.

5 Citado en Schreiner (2000).

6 De hecho, en la actualidad, las posibilidades de acceso al crédito formal por parte de microempresas bien podrían considerarse nulas. 
En efecto, el avance de este sistema de intercambio alcanzó niveles extraordinarios en el caso argentino y a través de su expansión se fue configurando una escisión social entre los que estaban dentro de la economía de mercado y los que estaban afuera (en el trueque). A medida que los individuos participan en él, su manejo de moneda de curso legal se reduce cada vez más. Entonces, al mismo tiempo que funciona como mecanismo de supervivencia, esta forma de intercambio empeora las perspectivas de retorno al sector monetario. De ahí que los microempresarios que solicitan créditos a instituciones de microfinanzas y venden sus productos en el mercado de trueque tengan serias dificultades para devolver los créditos.

\section{La población objetivo de las microfinanzas}

Existen dos grandes grupos poblacionales que podrían ser considerados como población objetivo o como potenciales demandantes de las microfinanzas en Argentina: los que están directamente excluidos del mercado de trabajo, es decir, los que forman parte de la desocupación abierta y los que participan en dicho mercado pero lo hacen de manera precaria. En esta última categoría incluiremos a los subocupados, y distinguiéndola de la población denominada informal.

Durante la década de 1990 la economía argentina sufrió fuertes transformaciones sociales de carácter negativo, desde el punto de vista de la distribución del ingreso, que tendieron a elevar a la población objetivo de las microfinanzas. En efecto, el porcentaje de la población que vive en áreas urbanas en condiciones de pobreza aumentó considerablemente, hasta alcanzar en octubre de $200257.5 \%$ (línea de pobreza) y 27.5\% (línea de indigencia), de acuerdo con las estadísticas oficiales del Instituto Nacional de Estadísticas y Censos (INDEC).

Además, desde el punto de vista financiero, la década de 1990 estuvo signada por la Ley de Convertibilidad (1991). En ese contexto la anulación del Banco Central como prestamista de última instancia creó la necesidad de imponer criterios regulatorios para el sector financiero mucho más exigentes que los sugeridos internacionalmente. Éste y otros factores elevaron las tasas de interés y tornaron más restrictivas las condiciones de acceso al crédito para los sectores menos protegidos de la economía.

La desocupación ha llegado a niveles sin precedentes. Pero tan importante como este fenómeno es el de la subocupación horaria, es decir, el de aquellos individuos que trabajan menos de una jornada completa. ${ }^{7}$ Dentro de estos últimos, se observa que el componente que más aumenta es el de los subocupados demandantes, es decir, de aquellos que se encuentran en condiciones precarias de trabajo y buscan activamente incrementar su jor-

La tasa de subocupación horaria es el porcentaje de ocupados, con respecto a la población económica mente activa (PEA) que trabajan menos de 35 horas semanales por causas involuntarias. Puede incluir a personas que hayan trabajado tan sólo una hora durante la semana en que se hizo la encuesta. 
nada laboral. Los niveles de desocupación y de subocupación horaria alcanzaron en octubre de $200217.9 \%$ y $19.9 \%$ respectivamente. $^{8}$

Asimismo, los niveles generales de informalidad ${ }^{9}$ que registra el país son altísimos. En Argentina se dispone de dos tipos de cifras de alcance nacional. ${ }^{10}$ La primera, y única de carácter oficial, es la que proviene del Sistema de Información, Monitoreo y Evaluación de Programas Sociales (sIEMPRo). ${ }^{11}$ La segunda, se dispone de las cifras de informalidad en Argentina publicadas por la oIT que muestra diferencias con las anteriores. Para el año 1997, las cifras de SIEMPRo alcanzan 39.9\% del empleo total, mientras que las de oIT arrojan $45.7 \%$. Pero de ambas se desprende que el nivel de informalidad alcanzado por la economía argentina era ya muy elevado en la década pasada.

\section{La situación de las IMfs en Argentina}

Esta sección está orientada a brindar un panorama sobre la situación de las IMFs que están operando en Argentina. Comenzaremos con una estimación acerca de la cantidad que se encuentra operando en el país. Luego presentaremos los resultados de un trabajo de campo realizado especialmente para este estudio, sobre quince IMFS ubicadas en distintas zonas.

\section{Las IMFS que actualmente operan en Argentina}

No existe, lamentablemente, un registro oficial de instituciones de microfinanzas. Esto hace muy difícil determinar con exactitud datos poblacionales básicos tales como cantidad de IMFs, total de créditos otorgados y monto de los mismos.

Por otro lado, es necesario aclarar que el papel financiero básico, casi exclusivo, de las IMFS en Argentina es otrogar microcréditos, dado que están imposibilitadas para captar depósitos. En efecto, no existe en la normativa del BCRA una categoría adecuada dentro el sistema financiero para este tipo de instituciones. Por eso las IMFS se conforman generalmente como organizaciones no gubernamentales (ONGS), lo cual representa una forma jurídica subóptima, ya que no las habilita para captar depósitos del público.

8 Dentro de los subocupados, aquellos que siguen siendo demandantes de trabajo alcanzaron $13.8 \%$ de la población activa (PEA) en octubre de 2002, según datos oficiales del INDEC.

9 El concepto de informalidad está ligado generalmente a personas pobres que deben insertarse en el mercado a través de estrategias de auto empleo de baja calificación y con un escaso nivel de capacitación, de capital inicial y de garantías para acceder al sistema financiero formal. Esto hace que sus actividades se limiten a la prestación de servicios no calificados, a pequeños comercios barriales o a la venta ambulatoria y en puestos fijos en ferias o mercados. Sus ingresos alcanzan generalmente sólo para la supervivencia familiar, lo cual dificulta el blanqueo de estos sectores.

10 Las mismas se basan en distintas metodologías y fuentes, pero aún así siguen la definición de la ort.

11 Este programa depende del Consejo Nacional de Coordinación de las Políticas Sociales de la Presidencia de la Nación y tiene a su cargo la denominada "Encuesta de Desarrollo Social, Condiciones de Vida y Acceso a programas y Servicios Sociales". 
En la Tabla 2 brindamos una estimación sobre la cantidad de instituciones que brindan servicios de microfinanzas, realizada sobre la base de un rastreo propio.

Tabla 2

Instituciones de microfinanzas que operan en Argentina

\begin{tabular}{lc}
\hline \multicolumn{1}{c}{ Categoría } & Cantidad \\
\hline IMFS & 35 \\
Total cooperativas & 7 \\
\hline Total & 42 \\
\hline
\end{tabular}

\section{Estudio de campo}

Al obtener información sobre el estado de las IMFs en Argentina, hemos realizado un estudio de campo que tuvo lugar durante el periodo julio-septiembre de 2002. Para ello, se contactó a la mayor parte de las instituciones detectadas ${ }^{12}$ con el propósito de realizarles una encuesta especialmente diseñada.

La muestra resultante está conformada por las quince instituciones que accedieron a responder a la encuesta y a suministrar información con mayor o menor grado de detalle. Cabe destacar que la misma incluye a las instituciones de microfinanzas más importantes dentro del ámbito no gubernamental. ${ }^{13}$

Para llevar adelante un análisis estilizado del comportamiento de las instituciones encuestadas, se procedió a clasificarlas en instituciones grandes, instituciones medianas e instituciones pequeñas. Con ese fin se tomó en cuenta la cantidad de beneficiarios que componen la cartera activa de las mismas según los siguientes valores:

- Instituciones grandes: desde 400 beneficiarios

- Instituciones medianas: entre 399 y 100 beneficiarios

- Instituciones pequeñas: menos de 99 beneficiarios

\section{Descripción de las IMFS de la muestra}

\section{Cartera activa y cartera histórica}

El primer punto que puede destacarse de la muestra es la cantidad de créditos y los valores otorgados por las instituciones. Estos datos son de suma importancia, dado que nos brindan un panorama general acerca del impacto que las microfinanzas tienen (cartera activa) y han tenido (cartera histórica) en Argentina.

12 Salvo el caso de las cooperativas, ya que en todos los casos consultados se nos informó que su actividad de microfinanzas era muy secundaria en relación con las otras actividades realizadas.

13 Los datos generales sobre las organizaciones encuestadas pueden ser observadas más adelante. 
Tabla 3

Cantidad de créditos y montos (\$ argentinos) otorgados según cartera histórica y cartera activa

Cartera histórica

\begin{tabular}{lrrrrr}
\hline & Cantidad & \% contrib. & \multicolumn{1}{c}{ Monto } & \% contrib. & $\begin{array}{c}\text { Monto promedio } \\
\text { de crédito* }\end{array}$ \\
\hline Grandes & 42504 & $91.5 \%$ & 101604983 & $98.8 \%$ & 2390 \\
Medianas & 2629 & $5.7 \%$ & 985620 & $1.0 \%$ & 375 \\
Chicas & 1300 & $2.8 \%$ & 232638 & $0.2 \%$ & 179 \\
\hline Totales & 46433 & $100.0 \%$ & 102823241 & $100.0 \%$ & 2214 \\
\hline
\end{tabular}

* Promedio agrupado de las IMFs (monto total/cantidad total)

Cartera activa

\begin{tabular}{lrrrrc}
\hline & Cantidad & \% contrib. & Monto & \% contrib. & $\begin{array}{c}\text { Monto promedio } \\
\text { de crédito }\end{array}$ \\
\hline Grandes & 8426 & $87.7 \%$ & 4596952 & $91.9 \%$ & 546 \\
Medianas & 954 & $9.9 \%$ & 369642 & $7.4 \%$ & 387 \\
Chicas & 228 & $2.4 \%$ & 37087 & $0.7 \%$ & 163 \\
\hline Totales & 9608 & $100 \%$ & 5003681 & $100.0 \%$ & 521 \\
\hline
\end{tabular}

* Promedio agrupado de las IMFs (monto total/cantidad total)

A partir de los datos obtenidos cabe destacar los siguientes aspectos:

- La escasa cantidad de créditos y de montos otorgados por las IMFs consideradas en la muestra, lo que corrobora el pequeño desarrollo que presentan estas instituciones en Argentina cuando se los compara con otros países del mundo y de América Latina.

- Al comparar los porcentajes de contribución de la cartera histórica con los de la activa, se observa que las instituciones medianas han acrecentado su participación.

- El fuerte peso relativo de las instituciones grandes (91.9\% de la cartera activa, por monto). Análogamente puede observarse que las IMFS pequeñas sólo colaboran con $2.4 \%$ de los beneficiarios (activos) totales y con sólo $0.7 \%$ de los montos activos prestados.

- La pronunciada disparidad de los montos prestados según el tamaño de las IMFS. El hecho de que el monto promedio de los créditos activos de las instituciones grandes sea más de tres veces superior al de las pequeñas puede estar reflejando la presencia de distintos perfiles de instituciones que apuntan, también, a diferentes mercados objetivos. Este fenómeno es aún más marcado en el caso de la cartera histórica.

- Al mismo tiempo se destaca una reducción, de casi 80\%, para el monto promedio de los créditos otorgados por las instituciones grandes (pasó de \$2 390 a \$546), mientras que el mismo se mantiene prácticamente constante para las medianas y para las pequeñas. 


\section{Antigüedad}

A pesar de que el sistema de microcréditos sea usualmente considerado como novedoso en Argentina, hay algunas IMFs que llevan más de diez años brindando servicio. Esto explica que la antigüedad promedio (considerada desde su fecha de creación hasta junio de 2002) de las IMFs encuestadas sea de 4.85 años (con un desvío estándar de 3.93 años).

Las instituciones presentan grandes variaciones en antigüedad de acuerdo con su tamaño. En efecto, mientras que las instituciones grandes tienen una edad promedio que ronda los diez años, las pequeñas y las medianas no alcanzan, en promedio, los tres años de vida. Esto puede explicarse, por el hecho de que una mayor perdurabilidad en el tiempo permite a las instituciones desarrollar una cartera más amplia de clientes. Del mismo modo, nos plantea interrogantes acerca del grado de perdurabilidad de las instituciones pequeñas y medianas.

\section{Servicios}

La prestación de servicios financieros muestra una significativa homogeneidad entre las distintas instituciones. Todas ofrecen microcréditos para sustentar actividades económicas (salvo una que los destina a la financiación de la construcción de viviendas propias) y casi el setenta y siete por ciento de las mismas ofrece servicios de capacitación (de diverso tipo).

En contraste con las tendencias que se observan en Asia y África, no se observa en Argentina una tendencia hacia la verticalización financiera de las IMFs. Por razones que ya fueron explicadas, ninguna de las instituciones consultadas ofrece el servicio de ahorro, contraviniendo la tendencia internacional de reemplazar el financiamiento externo con el citado servicio.

Es interesante comentar la categoría otros que compone la oferta de servicios de las IMFS consultadas. Más de $26 \%$ de las IMFs tiene una oferta de servicios que va más allá del crédito y la capacitación, llegando a ofrecer apoyo legal, psicológico, comercial. Como caso anecdótico podemos citar la prestación de servicios de pediatría a los clientes (por ejemplo las instituciones más pequeñas e informales, como las que surgen espontáneamente de las parroquias). Algunas IMFS toman a las microfinanzas como una manera alternativa de ayudar a la gente con sus necesidades, más que como un desarrollo profesionalizado de la práctica de las mismas.

\section{Tipo de préstamos y tasas de interés}

Aunque el Banco Central (BCRA) no considere a la garantía solidaria como una garantía válida, $40 \%$ de las IMFs consultadas trabaja exclusivamente con los denominados préstamos solidarios (grupales), con un promedio de 3.3 individuos por grupo. $27 \%$ de las IMFS 


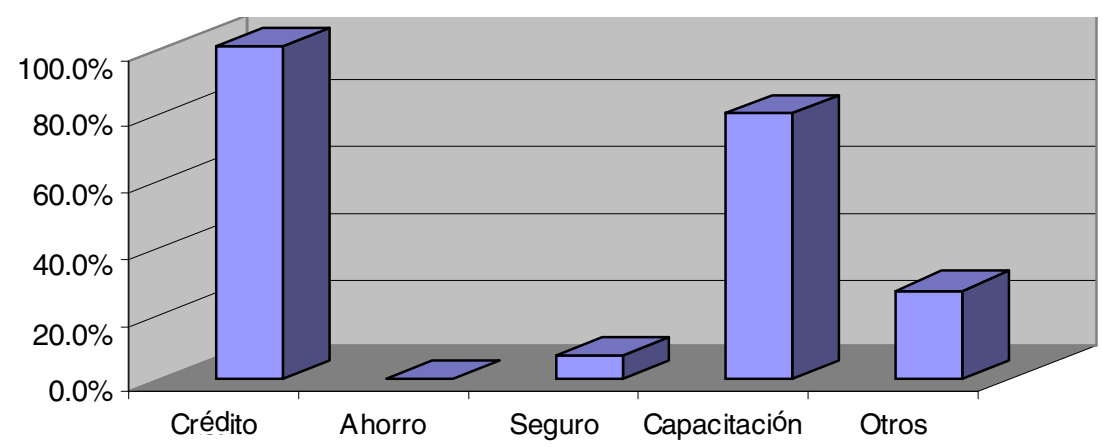

Gráfica 1. Servicios de las IMFS.

combina los créditos solidarios con el método de créditos individuales, mientras que 33\% restante otorga créditos exclusivamente individuales.

Con respecto a las tasas de interés, se detectó una gran heterogeneidad, entre las distintas IMFS, en cuanto a los factores que determinan los niveles de la misma (montos, plazos, comisiones, formas de devolución, son algunos de ellos).

De aquellas instituciones que presentaron datos sobre la tasa nominal anual obtuvimos un promedio de $37 \%$ con una elevada dispersión. Empero, debido a las metodologías particulares de reembolsos periódicos, y a la existencia de ciertos costos encubiertos, como en algunos casos la creación de un método de ahorro forzoso (el cual descuenta un porcentaje del monto percibido por el beneficiario como una especie de fondo de garantía a ser reintegrado luego del pago de la totalidad del crédito), las tasas efectivas suelen distar ampliamente de la cifra mencionada. De hecho, $40 \%$ de las instituciones cobra algún tipo de costo asociado al crédito: dentro de los que se encuentran gastos administrativos, gastos de capacitación, costos bancarios que son transferidos a los prestatarios.

\section{Objetivos}

A diferencia de lo que ocurre en otros países, ninguna de las instituciones encuestadas en Argentina apunta a obtener beneficios económicos, ya que las instituciones locales persiguen fines exclusivamente sociales. Sin embargo, dentro de este contexto hay diferencias en cuanto a los ejes fundamentales que hacen las instituciones para conseguir dichos fines.

Dentro de los objetivos planteados por las instituciones consideradas, hay propósitos compartidos (por $86.7 \%$ de las mismas) de facilitar el acceso al crédito a los sectores excluidos del sistema financiero formal y de contribuir a la reducción de la pobreza (80\%).

$60 \%$ de las IMFs plantearon otros objetivos, ligados a mejorar la calidad de vida de los beneficiarios, brindar confianza, generar trabajo a través del autoempleo, mejorar el proceso de comercialización de los bienes producidos por los microempresarios, entre otros. 


\section{Actividades de los microemprendedores}

La mayor parte de los emprendimientos respaldados (50.8\%), se dedica a actividades vinculadas al comercio. Cifras menores corresponden a las actividades de producción y servicios $(15.62 \%$ y $7.31 \%$, respectivamente).

\section{Escala y profundidad de las microfinanzas}

Un aspecto que puede resultar relevante es analizar el posicionamiento de las IMFS argentinas (explícito o implícito) en el debate sobre la escala y profundidad de las microfinanzas, es decir, si el objetivo de lograr el máximo impacto sobre la pobreza se logra priorizando la cantidad de clientes a los que se llega o su nivel relativo de pobreza (véase el apartado El trade off entre alcance y autosustentabilidad).

En ese sentido es importante destacar las respuestas de las instituciones tomando en cuenta su tamaño. Resulta así que $100 \%$ de las instituciones grandes prioriza maximizar la escala de los préstamos antes que la profundidad, mientras que $60 \%$ de las instituciones pequeñas tiene un comportamiento inverso, es decir, que le da prioridad a la llegada a los más pobres por sobre la escala (cantidad de préstamos). Este hecho podría dar cuenta de la existencia de papeles complementarios entre las instituciones pequeñas y las grandes, dado que cada una apuntaría a un mercado objetivo distinto. A continuación se presentan los datos sobre los créditos otorgados.

Tabla 4

Créditos otorgados por las IMFs encuestadas (monto en $\$$ argentinos)

\begin{tabular}{lrr}
\hline \multicolumn{1}{c}{ Datos históricos } & Cantidad & \multicolumn{1}{c}{ Monto } \\
\hline Primeros créditos & 6803 & 7170821 \\
Creditos a renovadores & 5849 & 10477199 \\
Sin categoría & 33781 & 85175221 \\
\hline Totales otorgados & 46433 & 102823241 \\
\hline
\end{tabular}

Como se observa, un total de 102 millones de pesos fue otorgado por concepto de préstamos por parte del conjunto de IMFS consultadas. Considerando que dicho monto se distribuyó entre 46433 créditos, se obtiene un promedio (histórico) de \$2 214 por préstamo. No cabe duda de que este monto es significativamente inferior a cualquier monto otorgado por el sistema bancario tradicional.

Lamentablemente, como algunas instituciones no tienen la información de primeros créditos, desglosada de la de renovaciones, no ha sido posible obtener (directamente de las fuentes) la cifra total de beneficiarios. Sin embargo, suponiendo una tasa igual entre los primeros créditos y los otorgados a renovadores para las IMFs que cuentan con esta informa- 
ción podemos obtener una proxy de la cantidad de beneficiarios. De acuerdo con este cálculo, serían 24967 las personas beneficiadas con microcréditos de las IMFs encuestadas.

Cuando desglosamos los valores de acuerdo con el tamaño de las instituciones observamos que las instituciones grandes (sólo cuatro IMFS) otorgaron alrededor de $90 \%$ del total de los créditos, tanto por cartera histórica como por cartera activa (véase Tabla 3). Es decir que, al hecho de que la escala de los microcréditos en Argentina sea muy baja (en comparación a otros países en desarrollo) podemos añadir que, además, dicha escala se encuentra altamente concentrada en unas pocas IMFs.

Por el lado de la profundidad, varios son los indicadores a tener en cuenta. En primer lugar, se encuentra el nivel de ingresos de los beneficiarios.

Tabla 5

Ingreso familiar de benificiarios

\begin{tabular}{lc}
\hline$<\$ 500$ mensuales & $33 \%$ \\
$>\$ 500$ mensuales & $67 \%$ \\
\hline Total & $100 \%$ \\
\hline
\end{tabular}

Esto es, 33\% de las personas que recibieron préstamos de alguna de las IMFS encuestadas están por debajo de la línea de ingreso mensual de los \$500.

Nuevamente, cuando desglosamos los valores de acuerdo con el tamaño de las instituciones, encontramos que las instituciones grandes y las pequeñas tienen papeles complementarios.

En efecto, existe una nítida segmentación del mercado objetivo según el nivel de ingresos familiares. Las instituciones pequeñas tienen $94 \%$ de clientes con ingresos mensuales inferiores a los \$500, mientras que las instituciones grandes destinan sólo $30 \%$ a dicha población. Por último, las instituciones medianas se acercan más al comportamiento de las instituciones pequeñas, pero casi $30 \%$ de sus beneficiarios tienen ingresos mensuales superiores a $\$ 500$. Estos datos complementan los de la Tabla 3, donde se mostraba una pronunciada disparidad de los montos promedio prestados según el tamaño de las IMFs.

\section{Conflictos entre consecución de los objetivos y sustentabilidad}

Otro aspecto que merece ser evaluado son los posibles conflictos entre el cumplimiento de los objetivos (como fueron definidos anteriormente) y la sustentabilidad. A continuación ofrecemos la percepción propia de las IMFS sobre este tema.

Más de la mitad las de instituciones consultadas (53.3\%) cree que la sustentabilidad financiera puede presentar conflictos con la consecución de los objetivos planteados por la institución. $40 \%$ considera que no existe tal conflicto (6.7\% no ha respondido la pregunta). 
Esto podría ser el reflejo de dos factores. En primer lugar, el asociado a las dificultades que presentan las IMFs para cobrar los créditos otorgados. En segundo lugar, a los costos operativos que suelen gravitar significativamente debido al bajo monto de los préstamos.

\section{Organización y funcionamiento de las IMFS}

\section{Tasa de productividad}

Si bien existen distintos instrumentos que pueden ser analizadas como variable proxy del éxito de las IMFs para funcionar en condiciones de eficiencia, la más sintética y elocuente es la productividad o tasa productiva. Ésta refleja la cantidad de clientes activos que cada organización soporta por empleado.

Como se observa a continuación, las cifras de productividad distan de ser buenas para las IMFs consultadas, dado que aproximadamente $40 \%$ de las mismas tiene menos de veinte clientes activos por empleado. Este dato cobra mayor dimensión cuando se observa que existen otras instituciones que tienen más de 100 clientes activos por empleado. El elevado desvío estándar (76.88 clientes) que presenta la media muestral (63.67 clientes) sugiere que, en muchos casos, las IMFs tienen un amplio margen para mejorar su nivel de eficiencia.

También es interesante observar los valores de la tasa de productividad para las distintas instituciones según su tamaño: 125.4 para las grandes, 29 para las medianas y 7.7 para las chicas.

Indudablemente, la diferencia de productividad según su tamaño es muy significativa y puede estar asociada a economías de escala en la provisión de microcréditos. Por otro lado las instituciones más pequeñas pueden estar conformadas por personal voluntario que trabaja sólo algunas horas a la semana, lo que permitiría explicar el hecho de que posean pocos clientes activos por empleado.

Cabe destacar que el indicador de productividad presenta algunas deficiencias. Una de las más importantes es que no mide la calidad de los servicios prestados. Por ejemplo, puede darse que una IMF con menos de veinte clientes activos por empleado preste a los mismos un servicio exhaustivo (capacitación, ayuda en la confección del plan de nego-
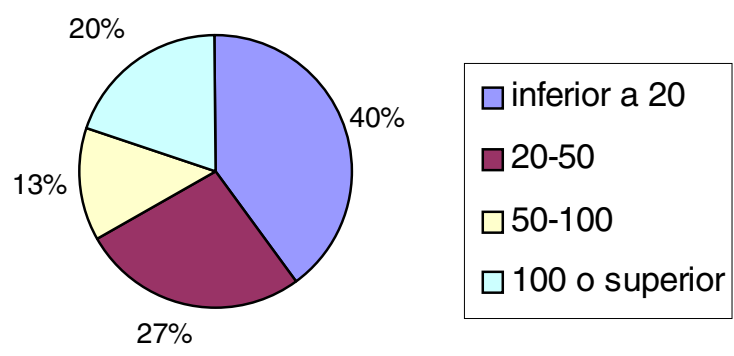

Gráfica 2. Tasa productiva. 
cios, seguimiento de cada paso, entre otros), mientras que una IMF con más de 100 clientes por empleado se limite a brindar únicamente el servicio básico (crédito).

\section{El empleo de incentivos dinámicos}

Un aspecto que nos parece de suma relevancia es que si bien $93 \%$ de las IMFs consultadas otorgan incentivos dinámicos a sus clientes, únicamente $20 \%$ de ellas hace lo propio con sus oficiales de crédito. Sin descartar otros factores, esto puede estar fuertemente vinculado al hecho de que una porción significativa de los empleados de las IMFs son voluntarios, lo cual dificulta la implantación de incentivos dinámicos.

\section{Comportamiento financiero de las IMFS}

Dos factores explican la dificultad de obtener tasas confiables y de carácter homogéneo sobre el comportamiento financiero de las IMFS. En primera instancia, el hecho de que las IMFS encuestadas no constituyan personería financiera hace que no se vean obligadas a tener un sistema de información sofisticado. En segundo término, no existe unanimidad acerca de las normas o principios más adecuados para el seguimiento financiero de las IMFS.

Ello dificulta obtener información para el armado de tasas conmensurables para las distintas instituciones. A continuación se exponen los resultados que han podido obtenerse en el marco de estas restricciones.

La tasa de recuperación acumulada (histórica) considera la totalidad de pagos recibidos sobre la totalidad de los vencidos a lo largo de toda la vida de una institución. Para las IMFS que poseen esta información (60\% de la muestra), la tasa de recuperación histórica es, en promedio, de $93 \%$ (con un desvío estándar de 7\%).

Nuestros datos arrojan que la cartera en riesgo 30 días (para $67 \%$ de la muestra) es de $22.9 \%$ en promedio. Dicho en otros términos, arriba de $20 \%$ del monto de la cartera activa de las IMFS presentadas se considera en riesgo de reembolso. Esta cifra denota un deterioro en la calidad de la cartera con respecto a la tasa de recuperación histórica previamente mencionada. Gran parte de las instituciones encuentran la respuesta a este empeoramiento en la profundo de la crisis que está atravesando el país.

Por último, la tasa de crecimiento (entre los años 2000 y 2001) de 53\% de las IMFS (para las que se poseen datos) es, en promedio, de 195\%. De este modo, se infiere que durante el año 2001 las IMFs transitaron por un periodo de gran expansión relacionada con su trayectoria previa.

\section{Recomendaciones de politicas}

El Estado tiene al alcance de la mano resolver importantes cuestiones que destrabarían el potencial de las microfinanzas en Argentina. A continuación ofrecemos una síntesis de recomendación de políticas. 
En primer término, sería altamente recomendable reestructurar el régimen impositivo para acoger la actividad de las microempresas. En particular, es necesario considerar la capacidad contributiva real de las mismas y sus especificidades en materia económica. En dicho régimen, la categoría más cercana a la capacidad contributiva de un microempresario (el monotributo) escapa totalmente a sus posibilidades, tanto por la inflexibilidad del sistema (supone pagos fijos y mensuales) como por la imposición de un monto mínimo fuera del alcance de la mayor parte de los microempresarios.

En segundo término, considerando la importancia de la venta de alimentos como actividad microeconómica, se requiere facilitar el acceso de las microempresas al control bromatológico de sus productos e instruir adecuadamente a las IMFs para que exijan la realización de dichos controles como condición sine qua non para el otorgamiento de microcréditos.

En tercer lugar, debe destacarse que no existe al momento en la normativa del Banco Central de la República Argentina (BCRA) una categoría adecuada para las instituciones que aspiran a brindar servicios microfinancieros. Estas instituciones están sujetas a los regímenes de aprobación y de supervisión del BCRA así como a su política de provisiones. Estos regímenes se vuelven prácticamente prohibitivos para la formalización de una IMF. La cantidad de requisitos, en términos de organización administrativa y funcional y de auditoría, incrementan los costos administrativos en forma incompatible con los bajos niveles de funcionamiento de las IMFs.

Asimismo, la normativa vigente en Argentina no considera la garantía solidaria como válida. Esto repercute sobre las instituciones financieras, y, finalmente, sobre quien recibe préstamos. La razón de ello estriba en que las garantías determinan los requisitos de provisiones que el BCRA obliga a mantener a las instituciones financieras. ${ }^{14}$

\section{Conclusiones}

El aumento de la población excluida del sistema económico, o precariamente integrada al mismo, que tuvo lugar durante los últimos años en la economía argentina, sumado al vacío provocado por la falta de acceso al crédito provisto por la banca formal, determinan la existencia de un mercado potencial importante para las microfinanzas. Sin embargo, las mismas constituyen en Argentina un fenómeno relativamente reciente y muestran un bajo grado de crecimiento cuando se las compara con otros países, tanto de Asia como de América Latina. Por otro lado, a diferencia de otras instituciones internacionales que están recurriendo en forma creciente a servicios de microahorro, las IMFS argentinas no han desarrollado este tipo de servicios por lo que sólo están vinculadas al sector de los microcréditos.

14 Es de destacarse que a diferencia del BCRA, el Banco Central de Bolivia ha estipulado como válida la garantía solidaria. Pero en el caso de que los créditos no estén debidamente garantizados, los bancos de dicho país sólo pueden dar créditos hasta un máximo de dos veces su patrimonio. 
A diferencia de lo sucedido en otros países, los microcréditos han tenido un desarrollo muy limitado en las zonas rurales de Argentina. En cambio, las urbanas presentan mejores condiciones para dicho crecimiento. La concentración de la población en estas zonas reduce los problemas derivados de la falta de mercado o de transporte. Asimismo, el aumento de la marginación ha flexibilizado de hecho el cumplimiento de las reglamentaciones que juegan en contra del desarrollo de las microempresas.

La principal restricción en cuanto a los microcréditos en Argentina se vincula con la falta de una oferta accesible y generalizada a las distintas áreas geográficas. A la escasa participación del estado en la implantación y promoción de las microfinanzas se agrega la existencia de un sistema bancario que ha estado tradicionalmente lejos de cumplir una función en ese sentido. Es más, los cambios sufridos por la economía argentina durante los años noventa llevaron a un proceso de concentración en la provisión del crédito a favor de los sectores de mayores recursos.

Respecto a la IMFS existentes en Argentina, la cantidad total que hemos rastreado, así como el nivel de los montos otorgados, nos muestran su carácter incipiente y el largo trecho que aún queda por recorrer. También apunta en esta dirección el estudio de campo realizado, en la medida que refleja un elevado margen para que las IMFs crezcan y mejoren su performance. Varios indicadores de nuestro estudio apoyan esta afirmación.

Para ello, sería necesario articular un esfuerzo conjunto de parte de distintos sectores de la sociedad. El aporte de donantes, del Estado y de los ámbitos universitario y tecnológico se vuelven clave para destrabar el mencionado potencial, pues de ellos dependerá el capital de trabajo, el marco regulatorio y las capacidades técnicas del sistema microempresarial.

En lo que concierne específicamente a la política pública, los puntos urgentes son la regulación de las microempresas y la habilitación de un mecanismo sencillo y de bajo costo con que las IMFS puedan contar para instituirse legalmente como tales.

La regulación de las microempresas presenta dos urgencias. La primera, garantizar el control bromatológico de las microempresas que comercializan alimentos. La segunda, proveer a los microempresarios de una opción impositiva que se ajuste a sus posibilidades e idiosincrasias. No sólo se perjudica el Estado (al no percibir impuestos) ante la actual carencia de un régimen impositivo adecuado para los microempresarios, sino también estos últimos, quienes se ven imposibilitados de transar en el mercado informal y obligados a lidiar con los controles policiacos y las inspecciones (que por su propia naturaleza buscan penalizar la actividad informal).

La regulación de las IMFS, por su cuenta, ha de comenzar por la promulgación de una personería jurídica que se amolde a su naturaleza y las deslinde de las costosas e inflexibles regulaciones del sistema financiero tradicional. Hasta que no se ofrezca una solución a este problema, las IMFS cargarán con los elevados costos de la informalidad, siendo entre ellos prominente la imposibilidad de tomar microahorros. 


\section{Bibliografía}

Bundell, Kevin, Microcredits and the needs of the poor, 1997.

Carbonetto, Daniel y Carbonetto, Sergio, Evaluación del Fondo MissionsProkur S.J. Crédito al sector del autoempleo urbano, Secretariado de Enlace de Comunidades Autogestionarias (SEDECA), 1996. , Sergio; Sandra Gioia y Marcos Solís, Microcréditos para el sector informal urbano, Cáritas Diocesana Quilmes, Cáritas, Comisión Nacional, Foro de Trabajo, 2001.

González-Vega, "Servicios financieros rurales: experiencias del pasado, enfoques de presente". Documento para el seminario El Reto de América Latina para el siglo XXI: servicios financieros en el área rural, La Paz, 1998.

INDEC, "Incidencia de la pobreza y de la indigencia en los aglomerados urbanos", Informe de prensa, Buenos Aires, abril, 2002.

Ledgerwood, Joanna, Manual de Microfinanzas, Banco Mundial, 1999.

Lescanne, Valerie, "Apostando al crecimiento. Evaluación del impacto de los servicios de apoyo a los microempresarios de menores recursos humanos", FONCAP, 1998

Morduch, Jonathan, "The microfinance schism", HIID Development Discussion Paper, núm. 626, 1996.

Parguez, A. y S.M. Seccareccia, "The credit theory of money: the monetary circuit approach", en Smithin J. (ed.), What is Money?, New York, Routledge, 2000.

Rahman, Sayeeda, "Microfinance: Helping the poor to Help Themselves", unESCO, 2000.

Schmitt, B., "A new paradigm for the determination of money prices", en Deleplace G. y E.J. Nell (eds.) Money in Motion. The Post-Keynesian and Circulation approaches, Mac Millan and St. Martin's Press, 1996.

Schreiner, Marl, Las microfinanzas en la zona rural de Argentina, Washington University, St. Louis, 2000.

SIEMPro, Encuesta de Desarrollo Social núm. 11. Trabajadores informales, Consejo Nacional de Coordinación de Políticas Sociales, Presidencia de la Nación, Buenos Aires, abril, 2001. 